\title{
Impact de la pollution urbaine sur la santé : le cas de la région parisienne
}

\author{
Myrto Valari $^{(1)}$, Laurent Menut ${ }^{(1)}$ et Édouard Chatignoux ${ }^{(2)}$ \\ (1) Institut Pierre-Simon Laplace, Laboratoire de météorologie dynamique, \\ CNRS UMR 8539, \\ École polytechnique - 91128 Palaiseau Cedex \\ (2) Observatoire régional de la santé d'île-de-France - Paris
}

\section{Résumé}

La météorologie et la composition atmosphérique en milieu urbain font l'objet d'une attention croissante. Les villes représentent les lieux où l'on trouve à la fois la plus grande densité de population et les plus fortes émissions de polluants gazeux et particulaires. La santé des personnes résidentes va donc être potentiellement dépendante de cette pollution locale urbaine et de sa capacité à se former et à se disperser. À l'heure actuelle, nous n'en sommes qu'au stade des premiers développements pour établir un lien entre pollution atmosphérique et santé. De nombreuses questions se posent : quelle est la part relative de la pollution atmosphérique extérieure vis-à-vis de tous les autres facteurs? Quelle est l'exposition réelle des personnes ? Peut-on vraiment différencier les effets particuliers de multiples polluants ? Pour répondre à ces questions, une chaîne de modélisation a été mise en place pour réaliser une première cartographie de l'exposition de la population de l'agglomération parisienne à l'ozone, au dioxyde d'azote et aux particules fines, et cela à une échelle horaire. Le modèle développé intègre un modèle météorologique, un modèle de chimie-transport et un calcul d'exposition. Il est montré que ces développements ont permis d'identifier de nouvelles associations épidémiologiques entre les niveaux ambiants de polluants et la mortalité en région parisienne.
D ans le cadre de la luttre contre la pollution atmosphérique, la première législation relative aux émissions industrielles est apparue en 1952 et a été motivée par le grand smog de Londres de la même année. Depuis, l'Organisation mondiale de la santé (World Health Organization en anglais, WHO en abrégé) a proposé des lignes directrices visant la surveillance de la qualité de l'air (WHO, 2005) et l'Union européenne a posé des objectifs à long terme avec le sixième programme d'action pour l'environnement (EAP $6^{\text {th }}$, 2007). À l'échelle européenne, des progrès conséquents ont été réalisés ces dernières décennies : globalement, les émissions ont été réduites, notamment pour le dioxyde de soufre $\left(\mathrm{SO}_{2}\right)$ et le plomb (Adams et Lukewill, 2010).

Les réductions sur les composés nitrés ont été moins importantes qu'on l'espérait et la pollution atmosphérique actuellement surveillée concerne principalement les particules (PM), l'ozone $\left(\mathrm{O}_{3}\right)$ et le dioxyde d'azote $\left(\mathrm{NO}_{2}\right)$. L'impact de ces polluants sur la santé est aujourd'hui évalué de façon très grossière : on considère une population homogène à l'échelle de l'agglomération sans pouvoir discerner le risque encouru par les individus en fonction de leur vulnérabilité particulière (démographie, santé, etc.). On considère également les polluants comme un ensemble homogène donc sans pouvoir quantifier la part relative des différents co-polluants. Enfin, des incertitudes importantes subsistent pour distinguer les effets sanitaires dus à une exposition chronique d'un impact à court terme.

En Europe, la principale source de pollution reste le secteur de l'énergie (70\% des émissions d'oxydes de soufre et $21 \%$ des émissions d'oxydes d'azote, $\mathrm{NO}_{\mathrm{x}}$ ). Mais le transport routier a aussi une part importante, puisqu'il est à l'origine de la majorité des émissions de monoxyde de carbone (CO), de $\mathrm{NO}_{\mathrm{x}}$ et de particules de diamètre inférieur à 2,5 $\mu \mathrm{m}\left(\mathrm{PM}_{2.5}\right)$. Ces polluants sont directement inhalés par les populations dans les grandes zones urbanisées.

\section{Les études épidémiologiques}

Ces dernières décennies, de nombreuses études épidémiologiques ont été menées pour corréler les concentrations de polluants à des problèmes de santé. Grâce aux études de suivi de cohortes (plusieurs centaines de personnes) au cours de longues périodes (plus de 10 ans), les effets à long terme de la pollution sur la santé sont aujourd'hui assez bien connus (Dockery et al., 1993 ; Pope, 2007). Cependant, l'impact à court terme de la pollution est bien plus difficile à évaluer. Cet impact fait partie d'un bilan complexe de la dégradation de la santé d'un individu et il est loin d'être le facteur le plus influent. Il faut en effet étudier d'autres paramètres, 


\section{Abstract}

Health impact of urban air-pollution: The case of the metropolitan Paris area

Urban meteorology and atmospheric composition has become an issue of great public concern. Megacities are areas of great population density and also of high rates of gaseous and particulate matter emission. The health of the residents of such large agglomerations will potentially be influenced by the local air-pollution, its formation and dispersion. Efforts are made to link air-pollution and health. Several questions are open: what is the relative contribution of the ambient air-pollution compared to all other exposure factors? What is the 'real' level of exposure reaching the individuals of a population? Can we distinguish the health effects of multiple-pollutants? To answer to these questions we have developed a modeling framework that maps the exposure of the Parisian population to ozone, nitrogen dioxide and fine particles at hourly resolution. This integrated model incorporates a meteorological model, a three-dimensional Eulerian air-quality model and a human exposure module. The first results of this modeling effort suggest new epidemiological evidence of the relationship between ambient airpollution levels and mortality over the city of Paris.

Le transport routier est une source de pollution importante. (C) Météo-France, P. Taburet)

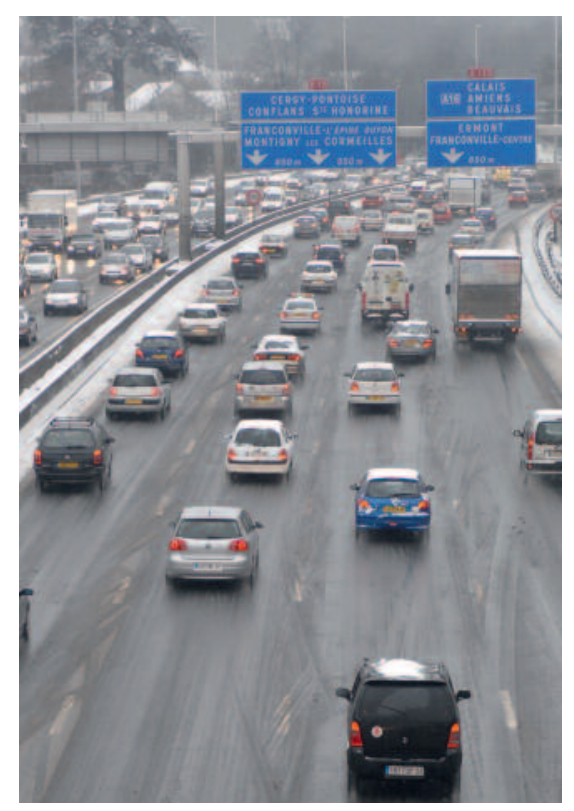

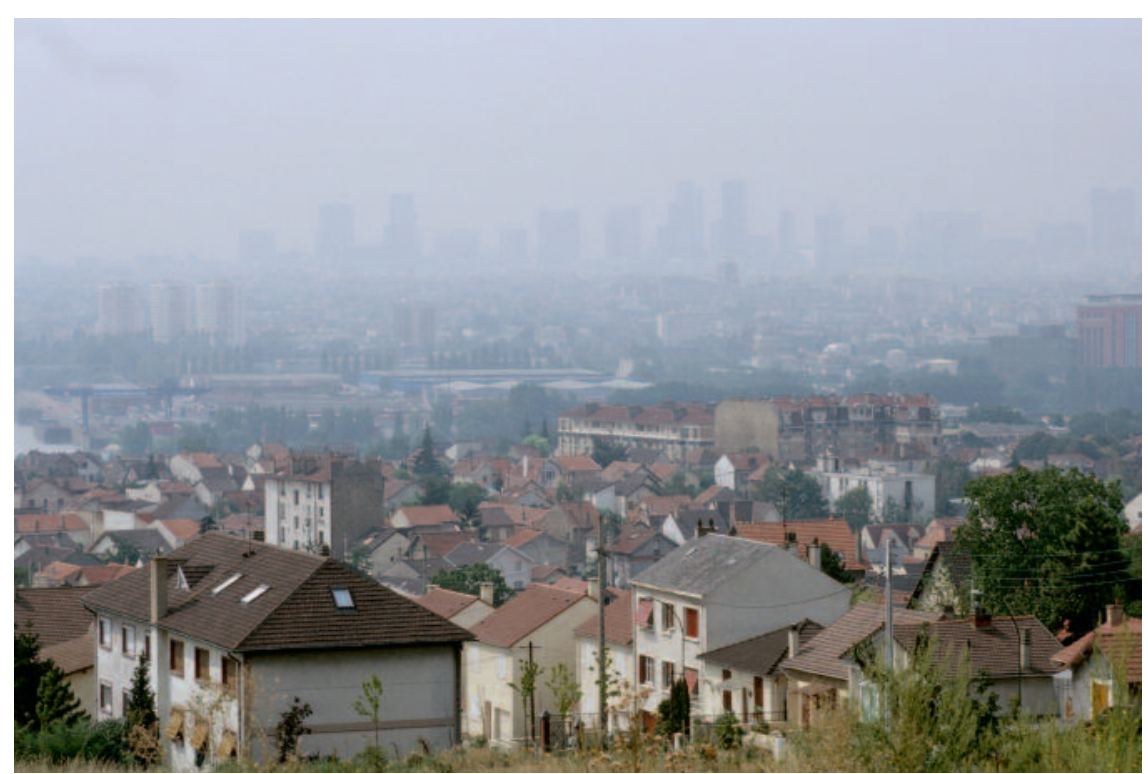

Pollution sur Paris. (C Météo-France, P. Taburet)

appelés co-facteurs (par exemples, conditions météorologiques et niveau socio-économique). Plus l'étude se rapproche de l'échelle de l'individu, plus les paramètres à considérer sont variés et rendent complexe la compréhension de ce bilan.

Les premières études d'impact à court terme ont été menées sur des échelles d'espace assez grandes avec des méthodes dites de séries temporelles. De manière générale, on cherche à associer les variations journalières d'un indicateur de pollution intégré sur une agglomération aux variations des données de santé agrégées sur cette même zone. Les tendances à long terme étant isolées (comme la saisonnalité), des relations statistiques peuvent être établies entre les niveaux de pollution journaliers et la mortalité. Des problèmes inhérents à ce type de modélisation ont été identifiés : des polluants atmosphériques, de nature chimique différente, peuvent avoir des effets distincts qui vont se compenser ou s'ajouter lors de l'évaluation du risque sanitaire. De plus, si la mortalité est un indicateur clair, il est difficile de recenser les personnes souffrant de la pollution qui ne sont pas hospitalisées ou qui ne se déclarent pas malades. Enfin, la méthode des séries temporelles permet d'estimer le nombre de cas attribuables à la pollution dans une sous-population vulnérable mais elle ne peut pas quantifier l'intervalle de temps pendant lequel un événement sanitaire a été anticipé ni la manière dont la pollution a affecté la population en la plaçant dans une catégorie à haut risque (on parle dans ce cas d'un « effet de moisson »).
Dans un premier temps, l'analyse de ce type de séries temporelles a été faite en comparant directement des séries chronologiques de concentrations de polluants et de mortalité ou morbidité (augmentation du risque de mortalité) [Samet et al., 1981 ; Ostro, 1984]. La mise en œuvre relativement simple et robuste de cette analyse en a fait une méthodologie de référence et de nombreuses études utilisent encore cette approche. Les recommandations des États en termes de niveau de pollution à respecter sont fondées sur ces méthodes (WHO, 2005). Des études plus récentes montrent que ces approches statistiques directes restent fiables (Dominici et al., 2002 ; Pope et Dockery, 2006). Toutefois les résultats obtenus restent agrégés sur des régions, sans distinction des polluants entre eux, et, surtout, sans spatialisation des résultats. Quelles sont les zones où la population est exposée à une pollution représentant un risque sanitaire sérieux ? Peut-on dissocier les différents pics de pollution (ozone, oxydes d'azote, particules) dans le signal global sur la santé ?

\section{Les modèles et données pour l'épidémiologie}

\section{Le principe du modèle de risque}

Dans le cadre de la présente étude, on va donc se focaliser sur des données de mortalité disponibles en région 


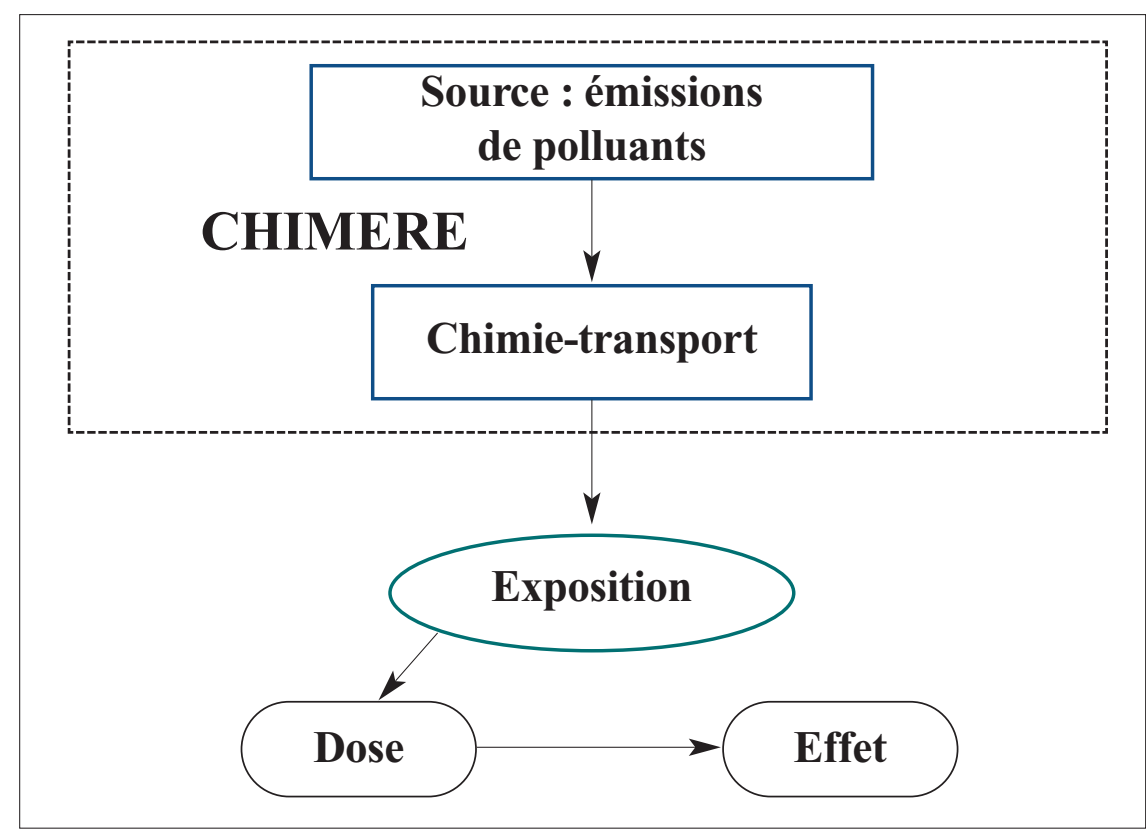

Figure 1 - Principe général du modèle de risque sanitaire.

parisienne. On cherchera à lever certains verrous importants :

- spatialiser le risque relatif lié à la population ;

- identifier les effets combinés des polluants en séparant leurs contributions ; - tenter de quantifier l'effet de moisson.

Pour cela, il faut construire un modèle de risque. Le principe est connu mais on va chercher ici à l'enrichir en informations. La figure 1 présente le principe général de ce modèle. Il est nécessaire d'évaluer les sources de polluants, puis de modéliser leur chimie et leur transport. Cet aspect est réalisé avec le modèle de chimie-transport, CHIMERE, développé au Laboratoire de météorologie dynamique (LMD) et à l'Institut national de l'environnement industriel et des risques (INERIS) [Bessagnet et al., 2008 ; Menut et Bessagnet, 2010]. Il faut ensuite estimer l'exposition : un modèle spécifique a été développé pour cette étude. Il faut quantifier la dose de polluant car la quantité « absorbée » sera différente des concentrations dans l'air. Enfin, il faut estimer l'effet des doses reçues par une population donnée.
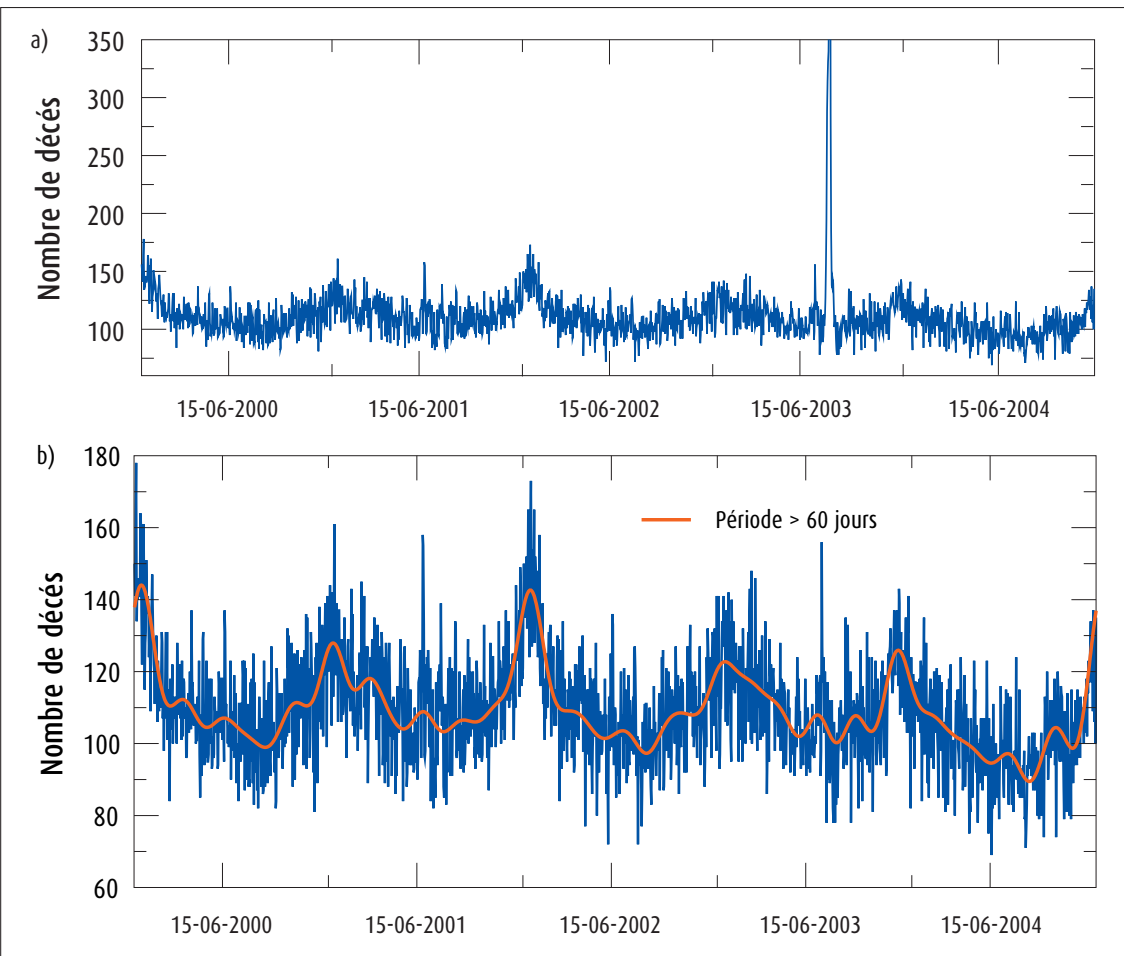

\section{Les données disponibles}

Les données disponibles pour la mortalité sont présentées sur la figure $2 \mathrm{a}$, pour la période d'étude allant de 2000 à 2004. On voit clairement un pic atypique lié à la canicule de l'été 2003. Afin de différencier les signaux en fonction de fréquences temporelles bien identifiées, une partie de l'étude va consister à supprimer les pics et à réaliser des filtrages temporels. La figure $2 b$ montre, par exemple, le résultat de la variabilité temporelle de la mortalité lorsque l'on supprime les fluctuations de périodes inférieures à 60 jours. On obtient alors, par différence, les effets à court terme de la pollution sur la mortalité. Ce principe est appliqué pour différentes fréquences, permettant de distinguer les impacts à court terme de ceux à plus long terme.

\section{La modélisation de la pollution atmosphérique régionale}

La première étape concerne la modélisation de la pollution atmosphérique en région parisienne. Afin de pouvoir se rapprocher des échelles réelles de l'exposition, il est nécessaire d'avoir une résolution spatiale et temporelle du modèle la plus fine possible : idéalement quelques mètres. La résolution du modèle est un choix de modélisation particulièrement sensible. En fonction de cette résolution, les émissions vont être plus ou moins diluées dans les mailles de calcul, pouvant ainsi modifier la chimie atmosphérique ellemême. Par exemple, la quantité d'ozone formé dépend des abondances relatives des émissions en composés organiques volatils et oxydes d'azote, qui définissent différents régimes chimiques.

Cette variabilité des résultats est présentée sur la figure 3. On quantifie l'impact de la résolution horizontale (ici en modifiant la résolution horizontale de la météorologie et des émissions) sur une même région et un même cas d'étude. La chimie de la pollution atmosphérique étant non-linéaire, il apparaît que

Figure 2 - a) Série chronologique du nombre de décès journaliers en région Île-de-France pour la période 2000-2004.

b) La période de canicule de l'été 2003 a été enlevée (du 22.08.2003 au 10.09.2003). La ligne rouge représente la même série après avoir ôté les fluctuations de périodes inférieures à 60 jours. 

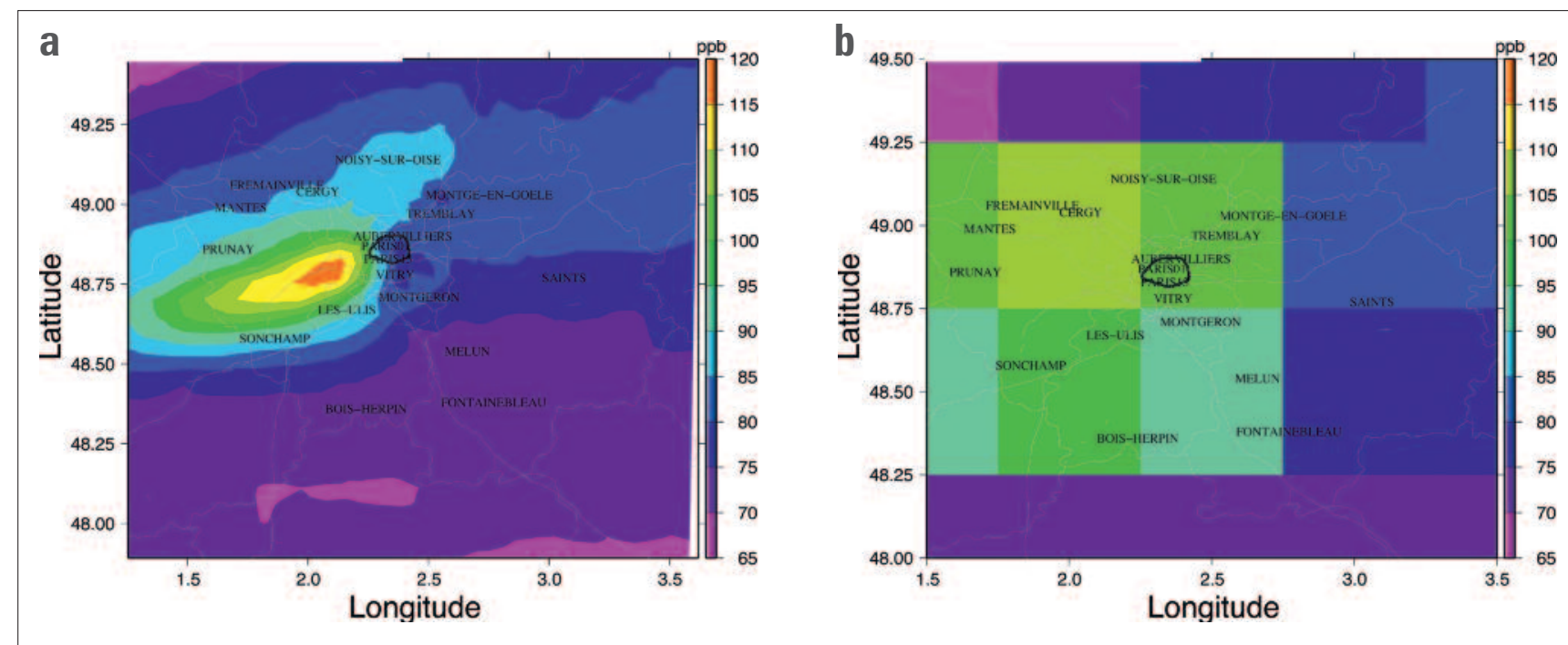

Figure 3 - Concentration en ozone de surface calculée avec le modèle CHIMERE au-dessus de la région Île-de-France pour le 3 août 2003 à 17 h 00 UTC. Les configurations du modèle sont : a) FINE et b) COARSE. Les paramètres météorologiques pour la simulation FINE sont calculés avec le modèle MM5 à $5 \times 5 \mathrm{~km}^{2}$ de résolution horizontale et les flux d'émissions sont diagnostiqués par l'inventaire d'AIRPARIF à $3 \times 3 \mathrm{~km}^{2}$. La simulation COARSE utilise des données météorologiques calculées avec MM5 à une résolution de 0,5 ×0,5 degrés et les émissions EMEP disponibles sur une grille de $50 \times 50 \mathrm{~km}^{2}$.

l'exposition estimée sera forcément dépendante de la résolution employée (Valari et Menut, 2008).

Afin de réaliser des statistiques fiables entre les valeurs de concentrations et les données de santé, il faut aussi simuler de longues séries temporelles de concentrations chimiques (plusieurs années). Idéalement, pour simuler des échelles spatiales de l'ordre de quelques mètres ou dizaines de mètres, il faudrait utiliser des modèles de type Large Eddy Simulation. Toutefois ces modèles ne permettent généralement que de simuler quelques rues et sur des événements de quelques jours.

Nous avons donc développé une nouvelle méthode, intermédiaire, permettant de calculer la variabilité sous-maille d'une espèce chimique avec le modèle CHIMERE. Cela permet de réaliser des simulations sur de longues périodes, tout en retrouvant des concentrations chimiques correctes mais sans explicitement réaliser les calculs à une résolution très fine. Le modèle a donc été utilisé avec une résolution spatiale de $5 \times 5 \mathrm{~km}^{2}$ et la méthode de variabilité sous-maille a été appliquée.

Cette méthode est construite à partir d'informations sur les émissions que les modèles n'utilisent pas en général. Dans un calcul classique de chimie-transport, on utilise la masse totale d'émission de chaque polluant sur une surface typiquement de l'ordre d'une dizaine de kilomètres carrés sur une ville. On perd alors l'information sur la provenance de cette espèce. Or la chimie urbaine rapide dépend fortement de la concentration des polluants lors de leur émission. Prenons l'exemple de l'oxyde d'azote (NO) qui est émis en large quantité par la circulation automobile sur des surfaces étroites (rues). Sa concentration locale au niveau de la rue sera bien plus élevée que celle du NO émis par des surfaces larges comme des zones résidentielles. En fonction des conditions météorologiques et des taux des réactions chimiques, il est probable que ce NO soit impliqué au sein de réactions chimiques très différentes, avant d'être globalement redistribué dans le volume complet de la maille du modèle. La méthode développée conserve cette information de secteurs d'activités lors du calcul de chimietransport en ajoutant une information sur les surfaces occupées par chacun de ces secteurs (surfaces des rues, parcs, usines, etc.). En couplant surfaces et secteurs, on est capable de simuler les concentrations des polluants localement dans des environnements urbains contrastés. L'ensemble des concentrations d'une même espèce est ensuite recombiné afin de calculer le transport vertical et horizontal, le mélange turbulent et le dépôt sec. La méthode a été optimisée et on ne calcule cette variabilité que pour les mailles hétérogènes en termes d'émission. Par exemple, on ne calcule pas une variabilité pour une maille constituée à $100 \%$ d'une forêt puisque celle-ci est considérée comme bien représentative de son environnement.

Cette méthode a été appliquée à la région parisienne. Elle a pu être validée sur des mailles où deux stations de surface du réseau AIRPARIF étaient disponibles (Valari et Menut, 2010). Un exemple de résultat est présenté sur la figure 4 . Il montre la comparaison entre deux types de mesures dans Paris, pour la même maille du modèle et pour des stations

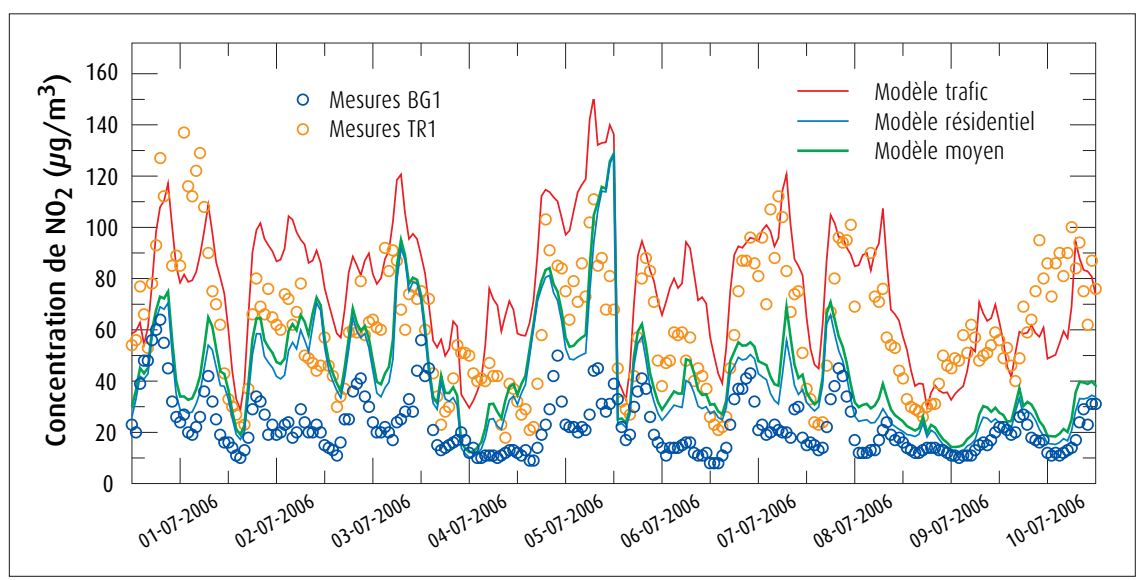

Figure 4 - Séries temporelles des concentrations de $\mathrm{NO}_{2}$ mesurées aux stations de type trafic (TR1) et de fond (BG1) du réseau AIRPARIF et modélisées avec CHIMERE. La courbe verte représente la valeur moyenne de la concentration $\mathrm{NO}_{2}$ modélisée avec le modèle de chimie-transport " classique ", alors que les courbes rouge et bleue correspondent au calcul sous-maille. 

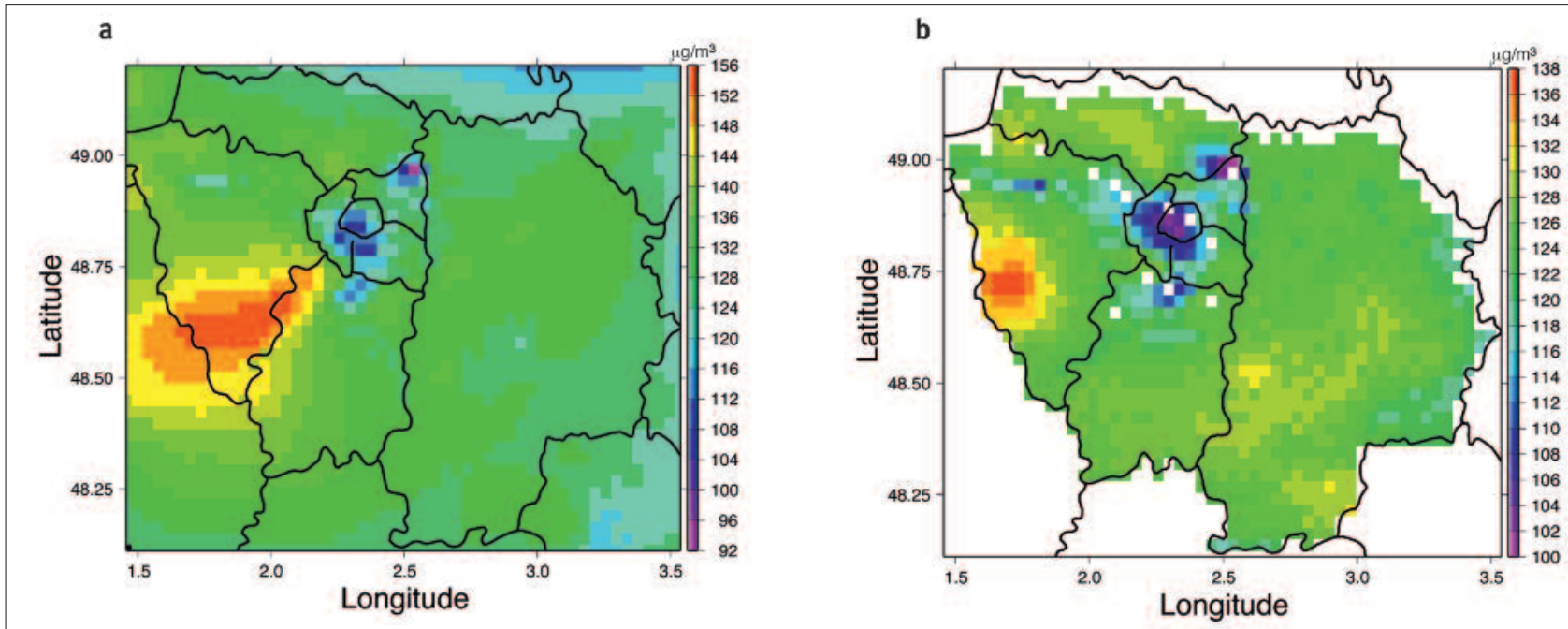

Figure 5 - a) Concentration d'ozone de surface en $\mu \mathrm{g} / \mathrm{m}^{3}$.

b) Expositions moyennes journalières à l'ozone de surface.

Résultats modélisés avec CHIMERE pour le 9 août 2003 sur la région Île-de-France.

trafic et de fond. Les symboles représentent les mesures. On peut voir que, pour le $\mathrm{NO}_{2}$, les concentrations horaires sont bien plus importantes pour la station proche du trafic que pour la station de fond. Par cette approche et pour les mailles les plus hétérogènes, on a pu calculer des distributions de concentrations pour l'ensemble des quatre années étudiées.

\section{Modélisation de l'exposition de la population}

Les études épidémiologiques récentes, comme InVS (2002), utilisaient un indicateur de pollution agrégé spatialement sur l'ensemble des stations de mesure disponibles dans la zone d'étude (réseau d'AIRPARIF pour la région Îlede-France). Pour améliorer la précision des études d'impact, nous avons développé un modèle qui :

- exploite la spatialisation accrue des champs de polluants modélisés avec le modèle de qualité de l'air CHIMERE; - prend en compte des facteurs d'exposition tels que la population et les déplacements domicile-travail ;

- calcule la distribution de l'exposition sur la région parisienne.

Ce modèle est fondé sur les données démographiques disponibles : le nombre d'habitants par départements franciliens, le pourcentage des personnes restant la journée dans le département, qu'elles travaillent ou non, ainsi que celles résidant dans un département mais travaillant dans un autre. Nous avons également utilisé des données précisant le temps passé dans les transports, au domicile ou au bureau par chacune de ces catégories de personnes.

Avec ce modèle de flux de population, il a été possible de quantifier une exposition finement spatialisée. En effet, les concentrations de polluants sont très variables à l'intérieur d'une région, comme le montre la figure $5 \mathrm{a}$ avec un panache d'ozone typique d'une journée estivale (ici l'été 2003). Ce panache se déplace sous l'effet du transport et va voir sa concentration se modifier en fonction des zones traversées et des temps caractéristiques de la chimie atmosphérique. De plus, il ne passera pas forcément sur des zones habitées. L'application du modèle d'exposition, combinée aux résultats de CHIMERE, montre l'exposition de la population aux polluants pour cette même date sur la figure $5 \mathrm{~b}$.

Pour chaque maille de la région et pour chaque polluant, cette exposition a été transformée en distributions d'exposition. Il est clairement apparu que trois modes principaux dominaient toutes les distributions calculées. Une paramétrisation a donc été développée pour convertir ces distributions en une exposition basse, moyenne et haute. Les résultats pour l'ensemble de la période et de la région sont présentés sur la figure 6 .

Ces résultats montrent que les habitants les plus urbains sont avant tout exposés au $\mathrm{NO}_{2}$ et aux $\mathrm{PM}_{25}$ (les sources de ces espèces primaires se trouvant en milieu urbain) alors que l'exposition la plus forte à l'ozone (espèce secondaire) est située en milieu rural (dans le panache). Si ce constat semble logique, la méthode a permis de quantifier avec précision les pourcentages relatifs de ces expositions. De plus, elle a aussi permis de calculer les expositions moyennes, généralement difficiles à estimer. La connaissance de ces contributions relatives permet alors d'utiliser un modèle de risque relatif qui les utilise comme données d'entrée.

\section{Modélisation de l'impact sur la santé}

\section{Le risque relatif}

Afin d'évaluer la méthode dans un contexte épidémiologique, nous avons utilisé un modèle de régression loglinéaire de Poisson (équation 1) :

$\log \left(\mu_{t}\right)=X_{t} \cdot \beta+S(t)+$ facteurs de confusion

où $\mu_{t}$ et $X_{t}$ sont le nombre de décès et un indicateur de la pollution pour le jour $t$. $\beta$ est ici la variable recherchée (l'inconnue de la régression) et exprime la relation entre pollution et mortalité. $S$ représente une fonction de lissage du temps $t$ et sert à modéliser la variabilité à long terme de la mortalité (saisonnalité). Les facteurs de confusion pris en compte ici sont les températures minimale et maximale du jour, les épidémies de grippe, le jour de la semaine et les vacances.

Pour étudier la sensibilité du modèle épidémiologique, la nouvelle approche est comparée aux études épidémiologiques précédemment publiées (par 


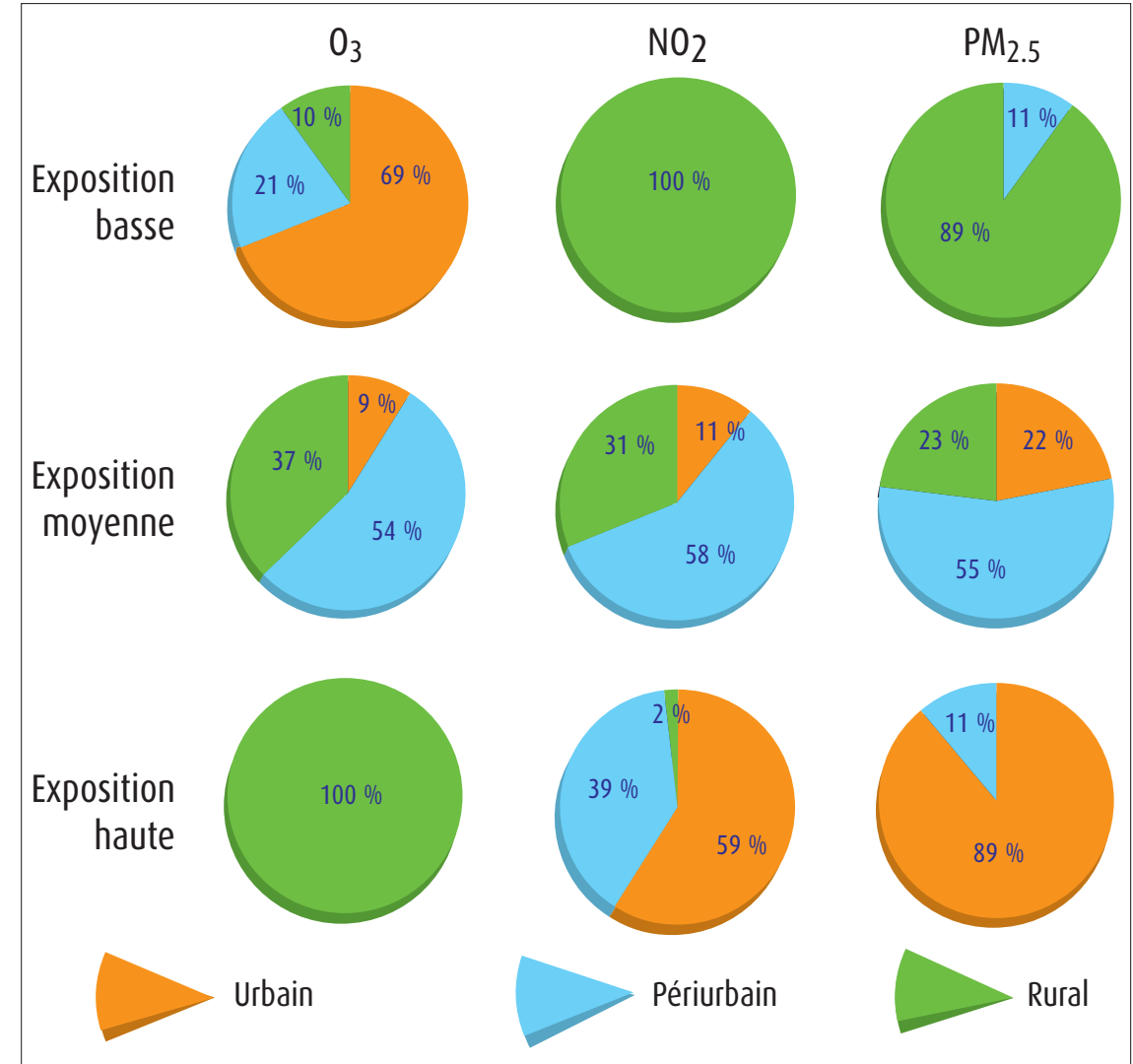

Figure 6 - Composition géographique des trois modes d'exposition à $\mathrm{O}_{3}, \mathrm{NO}_{2}$ et $\mathrm{PM}_{2.5}$ en zones urbaine (orange), périurbaine (bleu) et rurale (vert).

exemples, InVS, 2002 ; Katsouyanni et al., 2001). Trois indicateurs d'exposition différents ont été mis en œuvre dans le modèle suivant l'équation 1 :

- OBS : il s'agit de l'indicateur d'exposition habituellement utilisé. Il est construit à partir d'une agrégation spatiale sur l'ensemble des stations de mesures dans le domaine de l'étude ;

- CHIMERE : cet indicateur est fondé sur le même concept d'agrégation spatiale que OBS mais les concentrations de polluants sont cette fois extraites du modèle de qualité de l'air CHIMERE ;

-EXPO : cet indicateur est le plus réaliste puisqu'il prend en compte les déplacements de la population.

Enfin, deux configurations différentes du modèle épidémiologique ont été utilisées, mono-polluant et multipolluants, afin d'évaluer l'apport de chaque approche (Valari et al., 2010).

Les résultats présentés dans le tableau 1 sont exprimés en termes d'excès de risque relatif de mortalité (ERR) associé à une augmentation de l'indicateur d'exposition de $10 \mu \mathrm{g} / \mathrm{m}^{3}$. Cette valeur de concentration est une norme et s'applique aux différentes espèces chimiques étudiées.

La première méthode dite monopolluant reprend les développements des études épidémiologiques précédentes. En pratique, les indicateurs d'exposition sont gérés séparément dans le modèle. Un coefficient de risque est alors établi pour chaque polluant, indépendamment des autres. Cette configuration est fondée sur l'hypothèse d'une atmosphère parfaitement mélangée et où la pollution est homogène. Sous cette hypothèse le polluant dans le modèle de risque ne représente le même modèle de régression (modèle multi-polluants). pas uniquement sa propre toxicité mais celle de l'ensemble des co-polluants présents dans l'air ambiant. Le risque est d'obtenir des associations positives pour des polluants inoffensifs du fait qu'ils se trouveraient dans l'atmosphère en forte corrélation avec d'autres polluants réellement toxiques. D'après le tableau 1, la comparaison des trois résultats des modèles mono-polluant montre que :

- l'utilisation du modèle de qualité de l'air CHIMERE à la place des données mesurées permet d'établir des associations positives et statistiquement significatives entre pollution et mortalité ;

- la prise en compte des facteurs d'exposition (population et déplacements) change la contribution relative de chaque polluant à l'impact global de la pollution (donc pas d'association entre $\mathrm{NO}_{2}$ et mortalité).

La seconde méthode, dite multipolluants, inclut les différents polluants comme co-facteurs dans la même régression. Cette configuration permet, en théorie, d'estimer des relations d'exposition au risque propres à chaque copolluant. Le tableau 1 donne les excès de risque relatif (ERR) estimés avec les trois modèles, OBS, CHIMERE et EXPO, dans le cas où les trois polluants sont ajoutés comme des co-variables dans le même modèle. Cette configuration, a priori plus réaliste que le modèle mono-polluant, permet d'étudier les effets des co-polluants. La sixième ligne du tableau 1 (multi-polluants) présente les résultats du modèle de référence (OBS). Ce modèle n'est pas capable de distinguer les effets des

Tableau 1 - Excès de risque relatif (ERR, en \%) et intervalles de confiance de probabilité $95 \%$ [IC $95 \%$ ] pour la mortalité associés à une augmentation de $10 \mu \mathrm{g} / \mathrm{m}^{3}$ du niveau de l'indicateur de pollution. Les indicateurs d'exposition à $\mathrm{PM}_{25}$, à NO${ }_{2}$ et à l'ozone sont considérés séparément dans le modèle de régression (modèle monopolluant). En revanche, les indicateurs d'exposition à $\mathrm{PM}_{2.5}$, à $\mathrm{NO}_{2}$ et à l'ozone sont introduits simultanément dans

\begin{tabular}{|c|c|c|c|c|c|c|}
\hline \multirow{2}{*}{ Modèle mono-polluant } & \multicolumn{2}{|c|}{$\mathbf{P M}_{2.5}$} & \multicolumn{2}{|c|}{$\mathrm{NO}_{2}$} & \multicolumn{2}{|c|}{$\mathbf{O}_{3}$} \\
\hline & ERR & $95 \%$ IC & ERR & $95 \%$ IC & ERR & $95 \%$ KC \\
\hline OBS & 1,2 & {$[0,2 ; 2,2]$} & 1,4 & {$[0,9 ; 1,9]$} & 0,6 & {$[0,1 ; 1,0]$} \\
\hline CHIMERE & 0,9 & {$[-0,5 ; 2,3]$} & 1,1 & {$[0,0 ; 2,3]$} & 0,4 & {$[0,0 ; 0,9]$} \\
\hline EXPO & 0,9 & {$[-0,2 ; 2,0]$} & 0,3 & {$[-0,4 ; 1,0]$} & 0,4 & {$[-0,4 ; 1,2]$} \\
\hline \multirow{2}{*}{ Modèle multi-polluants } & \multicolumn{2}{|c|}{$\mathrm{PM}_{2.5}$} & \multicolumn{2}{|c|}{$\mathrm{NO}_{2}$} & \multicolumn{2}{|c|}{$\mathbf{O}_{3}$} \\
\hline & ERR & $95 \%$ IC & ERR & $95 \%$ IC & ERR & $95 \%$ IC \\
\hline OBS & $-1,1$ & {$[-2,6 ; 0,5]$} & 1,9 & {$[1,1 ; 2,7]$} & 0,4 & {$[0,0 ; 0,9]$} \\
\hline CHIMERE & 0,2 & {$[-0,5 ; 1,0]$} & 1,4 & {$[-0,3 ; 3,1]$} & 1,6 & {$[0,1 ; 3,1]$} \\
\hline EXPO & 1,3 & {$[-0,0 ; 2,7]$} & 0,4 & {$[-0,6 ; 1,4]$} & 0,9 & {$[-0,4 ; 1,2]$} \\
\hline
\end{tabular}


différents polluants. Une association négative est estimée entre les niveaux de PM et la mortalité alors que l'association entre $\mathrm{NO}_{2}$ et mortalité est bien plus élevée que dans le modèle monopolluant. Ce résultat, contraire à celui des études toxicologiques précédentes, montre que $\mathrm{NO}_{2}$ agit dans ce cas comme un substitut (proxy) de la pollution particulaire. En milieu urbain, $\mathrm{NO}_{2}$ et les particules fines sont principalement engendrés par la circulation automobile (combustion diésel). La corrélation entre les deux est tellement forte que l'on peut considérer $\mathrm{NO}_{2}$ et les particules fines comme des indicateurs globaux de l'exposition au trafic, qui est l'exposition majeure dans l'agglomération parisienne. Or, $\mathrm{NO}_{2}$ est mesuré par dix fois plus de stations que PM. Cela conduit à la situation paradoxale où les concentrations de $\mathrm{NO}_{2}$ représentent mieux l'exposition humaine aux particules fines que la mesure de ces derniers (Brook et al., 2007).

Au contraire, le modèle EXPO ne présente pas ce problème. Les associations sont positives et significatives pour les trois polluants. Cette représentation est plus réaliste, si l'on se réfère aux études en laboratoire ou sur des cohortes ${ }^{(1)}$. Ce résultat suggère qu'en ayant pris en compte des facteurs d'exposition réalistes (population et déplacements), le calcul d'exposition n'est plus autant limité par la représentativité des mesures utilisées. Cela permet de quantifier la part relative des différents co-polluants et d'obtenir des améliorations notables par rapport aux résultats actuellement disponibles dans la littérature.

\section{Les effets temporels}

Le dernier point étudié ici est une analyse des effets temporels sur l'association épidémiologique entre pollution atmosphérique et mortalité. Avec une décomposition en ondelettes de la série temporelle de pollution, on a créé une base orthogonale de six variables de pollution correspondant à six échelles d'exposition différentes. L'analyse statistique présentée précédemment (équation 1) est modifiée ici pour inclure les différentes composantes de la série d'exposition et modéliser des risques relatifs de mortalité propres aux différentes échelles temporelles. Cela est représenté par l'équation 2 :

$\log \left(\mu_{t}\right)=\Sigma X_{k t} \cdot \beta_{k}+S(t)+$ facteurs de confusion

(1) C'est-à-dire des statistiques épidémiologiques menées sur des milliers d'individus. où $k$ représente les échelles discrètes de la décomposition. Des moyennes journalières de mesures de surface de $\mathrm{NO}_{2}$ pour la période 2000-2004 ont été utilisées comme indicateur de l'exposition à la pollution urbaine. Les six variables indépendantes (résultantes de la décomposition de la série de $\mathrm{NO}_{2}$ ) sont utilisées comme co-variables dans le modèle résultant de l'équation 2 pour estimer les risques relatifs propres aux différentes échelles temporelles. Comme le montre la figure 7, l'analyse spectrale permet de mettre en évidence des effets sanitaires différents pour des échelles de temps courtes (inférieures à 15 jours) et intermédiaires (entre 15 et 55 jours). Pour les échelles de temps courtes, l'association épidémiologique reflète principalement un impact sur une sous-population à la santé déjà très fragilisée par des conditions chroniques. Le risque relatif décroît avec la durée de l'exposition car cette sous-population est de plus en plus épuisée (le principe de l'effet de moisson). En revanche, aux échelles intermédiaires, le risque relatif augmente à nouveau et a des valeurs positives. Cela reflète un impact sur la population générale qui devient vulnérable aux effets de la pollution et rentre dans la catégorie de sous-population à haut risque (Valari et al., 2011).

\section{Conclusion}

Le but de ce travail était d'établir un premier lien entre études épidémiologiques et modélisation déterministe de la météorologie et de la pollution régionale. Les relations existantes sont, pour la plupart, fondées sur l'analyse de concentrations mesurées de polluants gazeux et particulaires. Ici, nous avons pu mettre en évidence les limitations de cette approche, notamment l'impossibilité de prendre en compte la variabilité spatiale des champs de concentrations et la variabilité des caractéristiques d'une population (notamment ses déplacements au cours d'une journée et dans une région). Nous avons ainsi montré qu'une analyse fondée sur un indicateur de qualité de l'air uniquement issu de mesures ne permet pas la quantification de la part relative des différents co-polluants dans le risque global de la pollution urbaine. Grâce à la modélisation, cette contribution relative de chaque polluant devient quantifiable.

Pour réaliser cette modélisation, un modèle de proximité a été développé au sein du modèle CHIMERE. Cela permet aujourd'hui de modéliser les niveaux des polluants près de sources de pollution telles que les grands axes routiers, les zones résidentielles ou industrielles. En couplant cette information à petite échelle avec des données démographiques, nous avons réalisé un calcul d'exposition et montré une forte hétérogénéité dans la distribution spatiale de l'exposition en région parisienne. En modifiant le modèle épidémiologique pour tenir compte de cette hétérogénéité, nous avons pu nous affranchir du problème de séparation des effets parmi les co-polluants. Cela

Figure 7 - Les effets des échelles de temps sur la mortalité. Le pourcentage de mortalité est exprimé en fonction des différences temporelles entre pics de concentrations de $\mathrm{NO}_{2}$ et pics de mortalité en région parisienne.

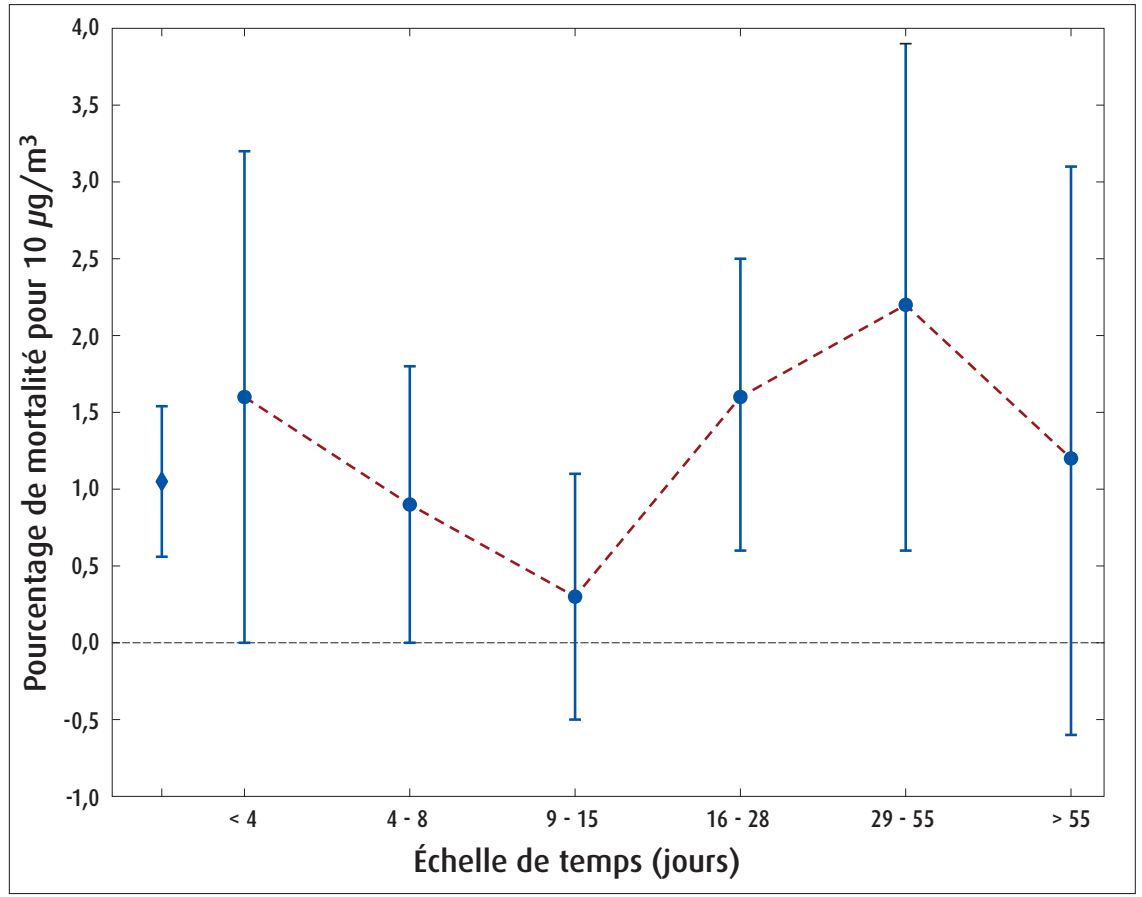


montre l'importance d'inclure des données comportementales et des calculs d'exposition dans ces études épidémiologiques.

Enfin, un développement purement épidémiologique a été effectué pour analyser les effets de la pollution à court terme sur la mortalité. Avec la méthode mise en place, nous avons mis en évidence le fait que la pollution n'affectait pas seulement les populations à haut risque de décès mais aussi une population plus générale, fragilisée par les événements de pollution urbaine.

Les perspectives de ces travaux sont tout d'abord d'étendre la méthode à d'autres régions et sur de plus grands domaines, comme la France. Un travail sur les données épidémiologiques devra être réalisé afin d'homogénéiser les bases de données disponibles. À terme, le calcul de la variabilité sous-maille et donc de l'exposition des populations viendra enrichir le site de prévision opérationnel PREVAIR (Rouil et al., 2009), utilisant déjà le modèle CHIMERE.

\section{Bibliographie}

\footnotetext{
- Adams M. et A. Lukewill, 2010 : The European Environment. State and outlook 2010. Air Pollution. European Environment Agency, Copenhagen, 1-42, www.eea.europa.eu/soer/europe/air-pollution.

- Bessagnet B., L. Menut, G. Aymoz, H. Chepfer et R. Vautard, 2008 : Modelling dust emissions and transport within Europe: the Ukraine march 2007 event. J. Geophys. Res., 113, D15202, D0I: 10.1029/2007JD009541.

- Brook J. R., R. T. Burnet, T. F. Dann, S. Cakmak, M. S. Goldberg, X. Fan et A. J. Wheeler, 2007 : Further interpretation of the acute effect of nitrogen dioxide observed in Canadian time-series studies. J. Expos. Sci. Environ. Epidemiol., 17 (S2), S36-S44.

Dockery D. W., C. A. Pope, X. Xu, J. D. Spengler, J. H. Ware, M. E. Fay, B. G. Ferris et F. E. Speizer, 1993 : An association between air pollution and mortality in - six U.S. Cities. N. Engl. J. Med., 329 (24), 1753-1759, www.nejm.org/doi/full/10.1056/NEJM199312093292401.

- Dominici F., A. McDermott, S. Zeger et J. Samet, 2002 : On the use of generalized additive models in time-series studies of air pollution and health. Am. J. Epidemiol. : 156, 193-203.

- EAP 6 ${ }^{\text {th }}, 2007$ : Mid-term review of the Sixth Community Environment Action Programme. http://ec.europa.eu/environment/newprg/review.htm, 1-17.

- InVS, 2002 : Programme de Surveillance Air et Santé 9 villes. Surveillance des effets sur la santé liés à la pollution atmosphérique en milieu urbain - Phase II. InVS, Saint: Maurice, 2002. www.invs.sante.fr/publications/2002/psas_020624/programme.html.

Katsouyanni K., G. Toulimi, E. Samoli, Y. Petasakis, A. Analtis, A. Le Tetre, D. Zminrou, F. Ballesret, A. Boumgnar, H. R. Anderson, B. Wojtyniak, A. Paldy,

- R. Braunstein, J. Pekkanen, C. Schindler et J. Schwartz, 2001 : Confounding and effect modification in the short-term effects of ambient particles on total mortality: - results from 29 European cities within the APHEA2 project. Epidemiology, 12 (5), 521-531.

: Menut L. et B. Bessagnet, 2010 : Atmospheric composition forecasting in Europe. Ann. Geophys., 28 (1), 61-74.

- Ostro B., 1984 : A search for a threshold in air pollution and mortality: a reanalysis of data on London winters. Environmental Health Perspectives, 58, 397-399.

- Pope C. A. et D. W. Dockery, 2006 : Health effects of fine particulate air pollution: lines that connect. Journal of the Air and Waste Management Association, 56, : 709-742.

- Pope C. A. III, 2007 : Mortality effects of longer term exposures to fine particulate air pollution: review of recent epidemiological evidence. Inhal. Toxicol., 19 (1), $33-38$.

- Rouil L., C. Honoré, B. Bessagnet, L. Majherbe, F. Meleux, R. Vautard, M. Beekmann, J.-M. Flaud, A. Dufour, D. Martin, A. Peuch, V.-H. Peuch,

- C. Elichegaray, N. Poisson et L. Menut, 2009 : Prev'air: an operational forecasting and mapping system for air quality in Europe. Bull. Amer. Meteor. Soc., 90, 73-83, - DOI: 10.1175/2008BAMS2390.1.

- Samet J., Y. Bishop, F. Speizer, J. Spengler et J. Ferris 1981 : The relationship between air pollution and emergency room visits in an industrial community. JAPCA, : 31, 236-241.

- Valari M. et L. Menut, 2008 : Does increase in air quality models resolution bring surface ozone concentrations closer to reality? Journal of Atmospheric and Oceanic - Technology, DOI: 10.1175/2008JTECHA1123.1.

- Valari M. et L. Menut, 2010 : Transferring the heterogeneity of surface emissions to variability in pollutant concentrations over urban areas through a chemistry transport - model. Atmos. Environ., 44, 3229-3238.

- Valari M., E. Chatignoux et L. Menut, 2010 : Using a chemistry transport model to account for the spatial variability of exposure-concentrations in epidemiologic air pol- Iution studies. Journal of the Air and Waste Management Association, en cours de publication.

- Valari M., L. Martinelli, E. Chatignoux, J. Crooks et V. Garcia, 2011 : Time scale effects in acute association between air-pollution and mortality. Geophys. Res. Lett., - en cours de révision.

: WHо, 2005 : Air Quality Guideline. Global update. World Health Organization, Regional Office for Europe.
} 